\title{
Soft X-ray Spectro-Ptychography for Air Pollution Particulates
}

\author{
Haigang Liu, Zijian Xu*, Chunpeng Wang, Xulei Tao, Yong Wang, Renzhong Tai
}

Shanghai Synchrotron Radiation Facility, Shanghai Institute of Applied Physics, CAS, Shanghai, China * Corresponding author: xuzijian@sinap.ac.cn

X-ray ptychography emerged in the late 2000s as a lensless imaging technique that combined the advantages of scanning transmission X-ray microscopy (STXM) and coherent diffractive imaging (CDI) [1]. Ptychography also overcomes the biggest disadvantages of both techniques: the limited resolution due to X-ray optics fabrication level for STXM, and the limitation to isolated objects and imaging size for CDI. The ptychography has been under rapid development since its first demonstration by hard Xray in 2007 [2]. Thereafter, quite a number of new ptychography algorithms were proposed and demonstrated by X-ray experiments [1,3]. With the rapid development and widespread availability of ptychography, many applications across various research areas have been explored. At present, ptychography has been applied in biology, chemistry, environment, material science and electronics. Recently, ptychography has been further developed in several advanced and highly interesting approaches, one of which is the spectro-ptychography [4]. This approach combines the high resolution of ptychography with the chemical specificity of spectroscopic X-ray techniques, which has great significance for element or chemical mapping analysis.

In this paper we show our research on STXM-based soft X-ray spectro-ptychography for air pollution particles imaging which will be helpful in revealing the possible sources of these pollutants and their potential toxicity to humans. In spectro-ptychography experiments, the incoming X-ray energy is varied across the absorption edge of an investigated element, while the incident beam is scanning across the sample and diffraction patterns are recorded for each scanning position and each energy point. A focused or defocused probe is chosen according to the sample scattering strength or sample size. It is also important to realize the automatic experiment by coordinating the data collecting system with the beamline monochromatic system. After data collection, how to process the data is the key issue of the spectro-ptychography, which includes: determining the diffraction pattern center; removing the background noise from the data; reconstructing sample images by a suitable algorithm which can remove some experimental illnesses (position drifting, beam incoherence, scanning grid, setup vibration, etc.); extracting the spectral information by a principal component analysis (PCA) software.

We performed a spectro-ptychography experiment for air pollutant particulates at Pohang Light Source. In this experiment, a defocused probe of 1 um diameter was used to scan across a dust particle with a step size of $200 \mathrm{~nm}$. The scanning grid was $15 \times 11$. The chosen energy range was $703 \sim 714 \mathrm{eV}$ with the energy step of $0.2 \mathrm{eV}$. The CCD exposure time for each scanning point was $250 \mathrm{~ms}$, and the total time of collecting data was about 85 minutes. After data collection, the data processing followed. First, the diffraction pattern center was determined by the Fresnel holographic area of the pattern. Then the background noise was removed by several newly designed techniques [5]. Though some new advanced algorithms can reconstruct the images better, the extended PIE (ePIE) was chosen for our data because ePIE is usually enough to reconstruct the sample images fast and well under coherent beam and precise scanning conditions. The spectral information was extracted by the following steps: 1) data converted to HDF5 format; 2) images alignment; 3) the spectral distributions were computed by a PCA software. Fig. 1 shows the reconstructed images and spectra analysis results. Some periodic mesh artifacts, though very 
weak, can be seen in these images, which should be the so-called raster grid pathology. A STXM image of the particle is also shown here, which looks much worse than the ptychography images. Power spectral density analyses indicate the STXM image has a resolution of $48 \mathrm{~nm}$ while the ptycho-images reach a resolution of about $15 \mathrm{~nm}$. From the spectral analysis results it can be seen that the content and valence of Fe element is varied across the whole sample, as the colored image shows. Combined with the cluster spectra, we can see that the most Fe-rich region (green) contains mainly ferric iron while other regions of the particle (yellow and red) seem to contain a mixture of ferric and ferrous iron. The analysis-derived image and spectra show the effective distribution of Fe element and its different valence states within the pollutant particulate, implicating its potential harmfulness to human health.

Our results suggest that the soft X-ray spectro-ptychography can be an effective tool to investigate the air pollution and environmental problems with high spatial resolution and spectroscopic resolution. In the following researches, we will use more advanced algorithms such as mixed state method to remove incoherent effects and further improve the reconstruction image quality for air pollutants.

References:

[1] F Pfeiffer, Nat. Photon. 12 (2018), 9-17.

[2] J.M. Rodenburg, A. C. Hurst, et al., Phys. Rev. Lett. 98 (2007), 034801

[3] P. Thibault, M. Dierolf and A. Menzel et al., Science 321 (2008), 379-382.

[4] M. Beckers, T. Senkbeil, T. Gorniak, et al., Phys. Rev.Lett. 107 (2011), 208101.

[5] C. P. Wang, Z. J. Xu, H. G. Liu, et al., Applied Optics, 56 (2017), 2099-2111.

[6] The authors thank Prof. Hyun-Joon Shin for his help in PAL. This work was supported by the National Natural Science Foundation of China (grant Nos. 11575283, 11505277, 11705273).
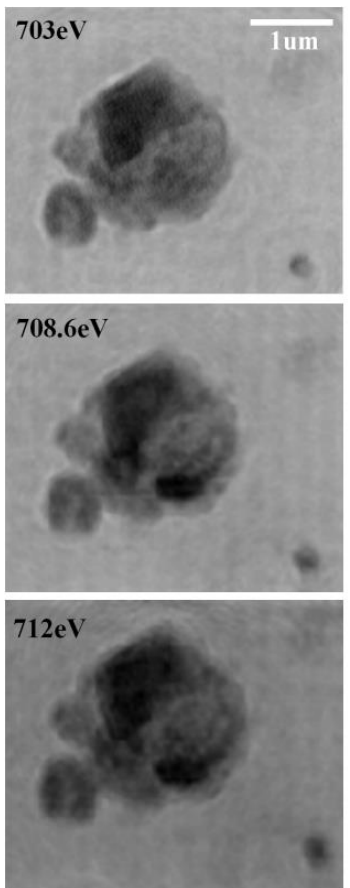
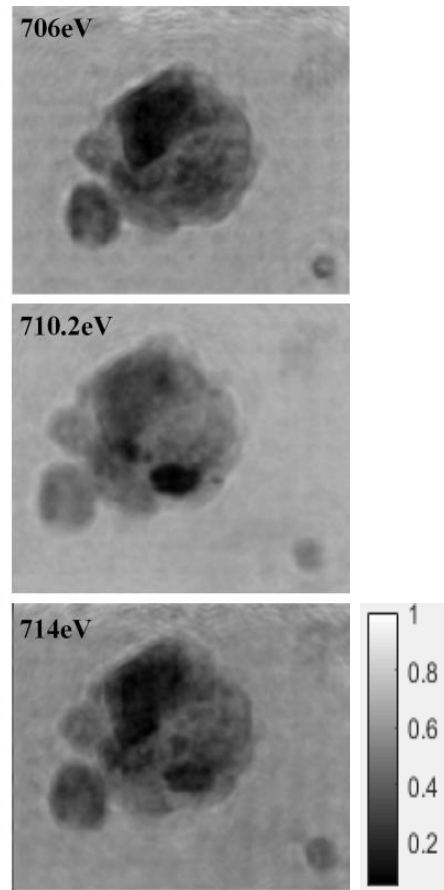
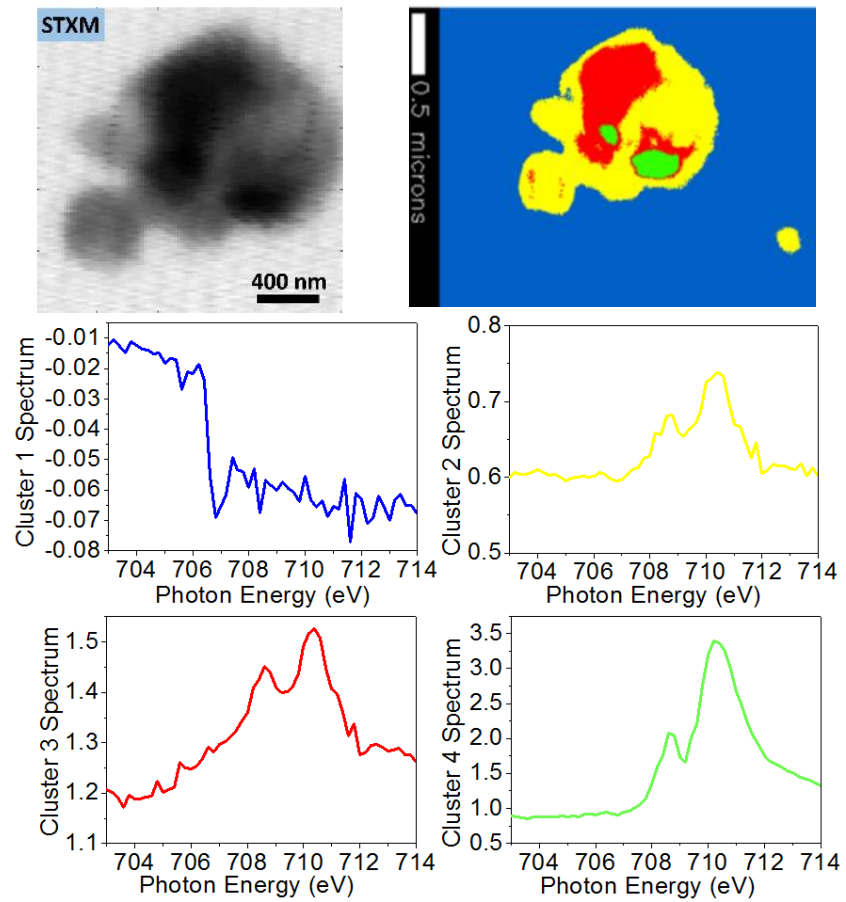

Figure. 1. Spectro-ptychography of an air pollutant dust. The images labeled with energy values are reconstructed sample amplitude images. A STXM image (labeled by "STXM", collected at $709 \mathrm{eV}$ ) with $20 \mathrm{~nm}$ steps is also shown for comparison. The colored image is the Fe chemical states distribution by PCA cluster analysis software, and the four line diagrams are the clustered spectra of Fe components for different areas of the sample. 